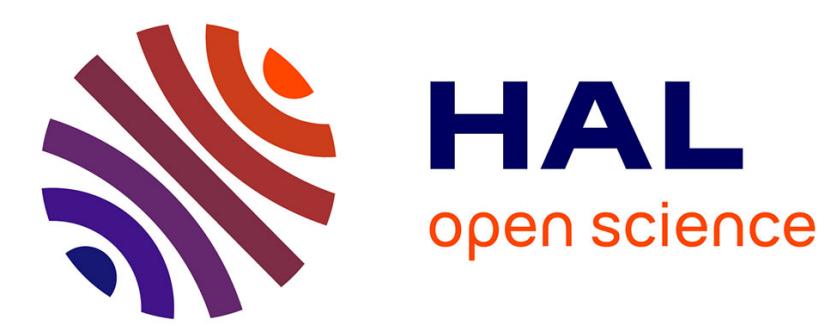

\title{
Channel estimation strategy for LPWA transmission at low SNR: application to Turbo-FSK
}

Vincent Berg, Jean-Baptiste Doré, Valérian Mannoni

\section{To cite this version:}

Vincent Berg, Jean-Baptiste Doré, Valérian Mannoni. Channel estimation strategy for LPWA transmission at low SNR: application to Turbo-FSK. VTC Spring 2019, Apr 2019, Kuala-Lumpur, Malaysia. cea-02293312

\section{HAL Id: cea-02293312 https://hal-cea.archives-ouvertes.fr/cea-02293312}

Submitted on 20 Sep 2019

HAL is a multi-disciplinary open access archive for the deposit and dissemination of scientific research documents, whether they are published or not. The documents may come from teaching and research institutions in France or abroad, or from public or private research centers.
L'archive ouverte pluridisciplinaire HAL, est destinée au dépôt et à la diffusion de documents scientifiques de niveau recherche, publiés ou non, émanant des établissements d'enseignement et de recherche français ou étrangers, des laboratoires publics ou privés. 


\title{
Channel estimation strategy for LPWA transmission at low SNR: application to Turbo-FSK
}

\author{
Vincent BERG, Jean-Baptiste DorÉ and Valérian MANNONI \\ CEA-Leti Minatec, 17 rue des Martyrs, 38054 Grenoble Cedex 9, France \\ \{vincent.berg;jean-baptiste.dore;valerian.mannoni\}@cea.fr
}

\begin{abstract}
Turbo Frequency Shift Keying has been considered as a promising physical layer for low power wide-area network connectivity. Because of its constant envelope amplitude and the efficiency of its iterative receiver performance close to Shannon's limit can be achieved. However, results published so far in the literature for the waveform have assumed perfect channel estimation or Signal-to-noise (SNR) levels that are higher than the SNR levels considered for these applications. This paper analyzes a channel estimation strategy based on a specifically adapted pilot sequence. Simulations have been performed to evaluate the performance of the proposed approach. Performance loss induced by imperfect channel estimation algorithms is estimated.
\end{abstract}

Keywords-LPWA; Zadoff-Chu; Constant Envelope; NB-IoT

\section{INTRODUCTION}

The installed base of the Internet of Things (IoT) devices is forecast to grow to almost 31 billion worldwide by 2020 . Longrange, wide-area networks (LPWA) including narrowband IoT (NB-IoT) are expected to make a significant part of the almost 2.6 billion Machine-to-Machine (M2M) connections that will be in place by that time [1].

For LPWA systems, low energy consumption requirement directly results from the necessity to have a long battery life. Long range is achieved with performance at a very low level of sensitivity at the receiver and obtained by ensuring Quality of Service (QoS) for low levels of Signal-to-Noise Ratio (SNR). At the physical layer, this is achieved by selecting an energy efficient modulation combined with an efficient usage of the Power Amplifier (PA), the most power-consuming component of the transmission chain [2]. The efficiency of the PA is highly dependent on the peak-to-average power ratio (PAPR) of the signal [3]. NB-IoT as defined in Release 13 of the 3GPP specification [4] considers both Single Carrier Frequency Division Multiplexing (SC-FDM) and single-tone transmission to limit peak-to-average power ratio (PAPR). The use of single tone transmission is particularly interesting for LPWA communications. Beside, this is not without any drawback. As the bandwidth is small, the transmission time duration is significantly increased in comparison to SC-FDM transmission and hence the energy to transmit an information bit. However, PAPR levels of SC-FDM is significantly larger than $\mathrm{OdB}$. For these reasons, alternatives to SC-FDM should be considered.

Turbo Frequency-Shift Keying (Turbo-FSK) is a new waveform introduced in [5] that combines a non-linear modulation with a convolutional code. Its receiver relies on turbo processing to meet a performance close to Shannon's limit for low spectral efficiency. Furthermore, the waveform exhibits a constant envelope (i.e.: its PAPR is equal to $0 \mathrm{~dB}$ ). Turbo-FSK is therefore well adapted to future LPWA systems notably for the 5 th generation of cellular systems.

The principles of Turbo-FSK have been first described in [5]. The motivation for the scheme was to operate close to the Shannon capacity while using a constant envelop modulation. The structure of the transmitter (Fig. 1) is composed of $\lambda$ repetition stages. For each stage, a differently interleaved version of the same input information bit sequence is encoded using a simple parity accumulator. The coded bits are then mapped onto a Turbo-FSK codeword: a combination of a linear $N_{L}$-point phase shift keying (PSK) modulation and a nonlinear $N_{\perp}$-carrier FSK modulation [6]. Efficient digital implementation can be realized by inverse Fast Fourier Transform (FFT) followed by a cyclic prefix insertion as for Orthogonal Frequency Division Multiplexing (Fig. 1). At the receiver, a soft FSK-detector estimates the probabilities of each possible Turbo-FSK codeword. These probabilities are then fed to the decoder, which uses them as channel observation, while output of the other decoders will be used as a priori information. A modified version of the algorithm proposed by Bahl, Cocke, Jelinek and Raviv (BCJR) [7] is used to decode the trellis, and derive the a posteriori probabilities of the information bits. The association of the encoding with a non-linear modulation (FSK) allows to operate at very low levels of SNR. It has been demonstrated that the choice of the PSK is the optimal modulation minimizing channel capacity gap to Shannon's limit [8]. Practical implementations of the FSK-detector consist of a FFT combined with a frequency domain equalizer that relies on accurate channel estimation.

Expected SNR at the receiver should be well below $0 \mathrm{~dB}$ and particularly since Turbo-FSK proposes to improve energy efficiency to within a few decibels of Shannon's limit, . A typical level of SNR at sensitivity limit is expected to be of around $-10 \mathrm{~dB}$ for LPWA applications. This imposes significant performance constraints on channel estimation at the receiver. Results published so far in the literature for the waveform have either assumed perfect synchronization and channel estimation or considered significantly higher levels of SNR [9]. This paper derives a channel estimation algorithm optimized for the very low levels of SNR required by low power IoT connectivity applications.

The paper is structured as follows: Section II presents a frame and pilot structure adapted to Turbo-FSK. In Section III, channel estimation algorithms are described and evaluated. Performance comparison against perfect channel estimation is given and an optimized structure is discussed. Section V concludes the paper. 


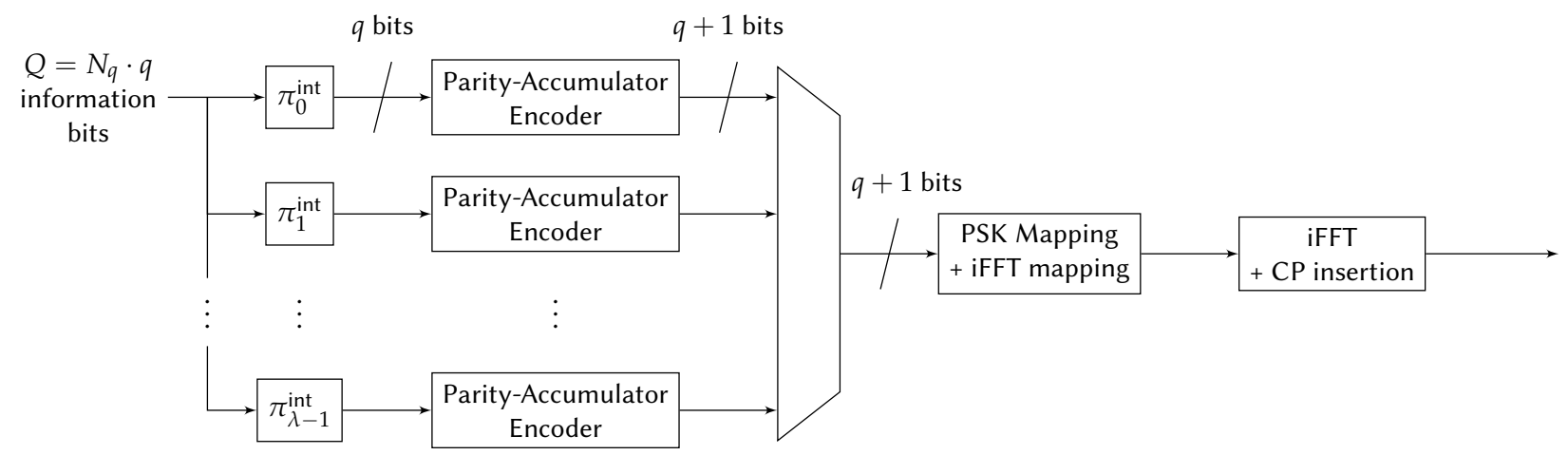

Fig. 1. Transmitter architecture of Turbo-FSK.

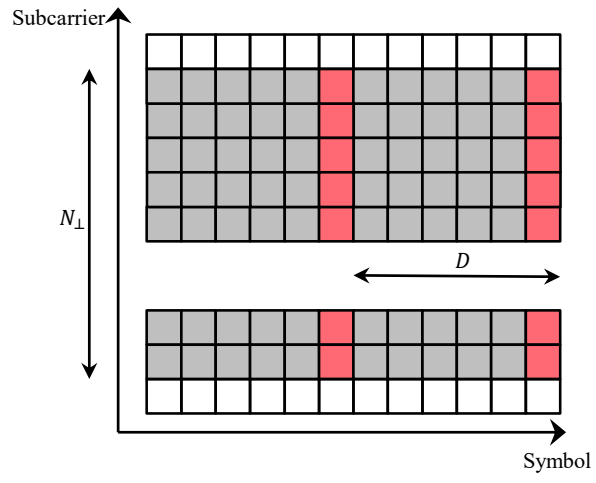

Fig. 2. Pilot structure of the frame

\section{System MODEL}

\section{A. Frame and Pilot Structure}

A typical LTE uplink frame structure is considered for channel estimation. The resource grid structure where $N_{\perp}$ subcarriers are active is composed of data symbols interleaved with pilot symbols spaced every $D$ symbols (Fig. 2). The structure is designed to give an estimate of the frequency response of the channel and to track its evolution during the duration of the transmission. In order to keep the lowpower advantage of the waveform, we imposed for the pilot symbol to have a low PAPR. As a consequence, constant amplitude, zero autocorrelation (CAZAC) sequences have been considered. Zadoff-Chu (ZC) sequences [10] are an example of such sequences and have already been widely used in cellular systems. Two approaches may be considered. For the first approach, the $\mathrm{ZC}$ sequence is generated in the time domain.

$$
z[k]=\alpha e^{-j \pi \frac{k^{2} N_{\perp}}{N^{2}}} e^{j 2 \pi \nu \frac{k}{N}}, \forall k \in[0, N]
$$

where $\alpha$ is a scrambling factor that is constant for each pilot symbol, i.e. $\alpha \in\{-1,1\}$. The parameter $N_{\perp}$ is the number of active carriers and $N$ the number of samples per symbol (the size of the FFT). The scalar $\nu$ is a normalized frequency offset used to shift the spectrum of the time sequence around the appropriate frequency resource block. A second approach considers the generation of the $\mathrm{ZC}$ sequences in the frequency domain as follows:

$$
Z[k]=\alpha e^{-j \pi \frac{k^{2}}{N_{\perp}}}, \forall k \in\left[0, N_{\perp}-1\right]
$$

TABLE I. ZC SEQUENCE COMPARISON WHEN $N=512$ AND $N_{\perp}=32$

\begin{tabular}{llll}
\hline & PAPR [dB] & $\begin{array}{l}\text { Adj. Leakage } \\
\left(N_{\perp}+1\right)[\mathrm{dB}]\end{array}$ & $\begin{array}{l}\text { Adj. Leakage } \\
\left(N_{\perp}+2\right)[\mathrm{dB}]\end{array}$ \\
\hline ZC sequence (z) & 0 & 17.3 & 40.8 \\
$\begin{array}{l}\text { ZC sequence (Z) } \\
\text { Modified }\end{array}$ & 2.6 & $<65.0$ & $<65.0$ \\
ZC sequence (Z), $\rho_{d B}=7$ & 0.7 & $<65.0$ & $<65.0$ \\
\hline
\end{tabular}

The ZC sequence of (2) is then placed on the allocated resource carriers and the associated time sequence is generated using a N-point inverse FFT. Both alternatives have advantages and drawbacks. The ZC sequence based on (1), also called time ZC sequence, provides a constant envelop signal but localization in the frequency domain is not optimal: frequency orthogonality is not ensured between synchronized users at least a guard interval of one frequency tone should be considered. For instant, up to $17.3 \mathrm{~dB}$ of energy is leaked to the out-of-band adjacent carrier (carrier $N_{\perp}+1$ ). On the other hand, the $\mathrm{ZC}$ sequence based on (2), also called frequency $\mathrm{ZC}$ sequence, is by construction orthogonal between different users using different resource blocks, however when upsampled by the inverse FFT transform, the PAPR of the generated sequence is significantly larger than $0 \mathrm{~dB}$. Table I gives a performance comparison of both sequences in terms of PAPR and adjacent channel orthogonality for $N_{\perp}=32$ and $N=512$ where adjacent channel power leaked on carriers $N_{\perp}+1$ and $N_{\perp}+2$ is given.

To improve the PAPR properties of the frequency $\mathrm{ZC}$ sequence, we designed a modified frequency $\mathrm{ZC}$ sequence composed of the ZC sequence of (2) and an additive noise vector $X$ selected in such a way that the resulting PAPR of the transmitted sequence is minimized and the signal-tointerference ratio (SIR) on each subcarrier is contained.

$$
Z_{M o d}[k]=Z[k]+X[k], \text { with } \frac{1}{|X[k]|^{2}}>\rho,
$$

$\forall k \in\left[0, N_{\perp}-1\right]$. The resulting PAPR and leakage performance of the modified ZC frequency sequence is given in Table I. Significant reduction of PAPR level is achieved: optimization gives a PAPR as low as $0.7 \mathrm{~dB}$ when the allowed signal-tointerference level is set to $7 \mathrm{~dB}$.

In order to evaluate the impact on the autocorrelation properties of the ZC sequence performance, the ambiguity function of the modified frequency $\mathrm{ZC}$ sequence has been evaluated and 

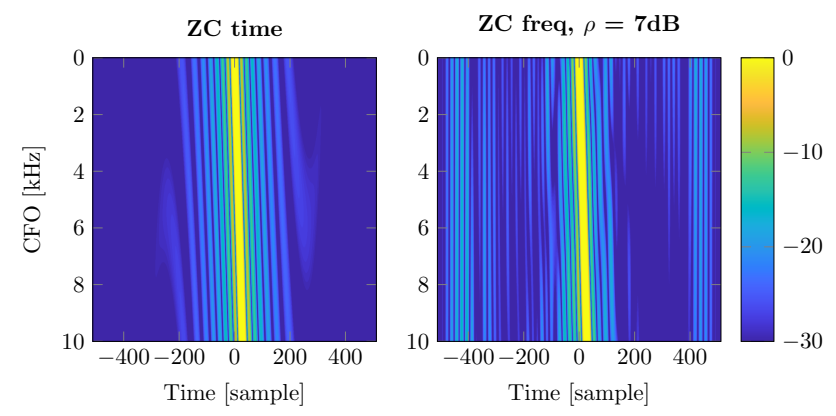

Fig. 3. Ambiguity function comparison. Left time ZC sequence as in (1) and right modified $\mathrm{ZC}$ frequency sequence as defined in (3)

compared to the time $\mathrm{ZC}$ sequence. The ambiguity function is defined as follows:

$$
\mathcal{A}(f, t)=\left\|\sum_{k} z^{*}[k-t] z[k] e^{j 2 \pi f t}\right\|^{2}
$$

Fig. 3 gives the ambiguity function for the modified $\mathrm{ZC}$ sequence when $\rho$ is set to $7 \mathrm{~dB}$ and compared to the time sequence. It demonstrates that the interference introduced to optimize PAPR has very little impact on the autocorrelation properties of the scheme and therefore has little impact on channel estimation performance.

\section{B. Channel Estimation}

The proposed pilot sequence is used to estimate the channel information state. A three step procedure is here considered. First, a least square (LS) estimation on the pilot subcarriers is performed. Then, a de-noising filter is applied on the $N_{\perp}$ LS estimates. Finally, channel estimation on pilot symbols is interpolated in the time domain to provide a channel estimate of each Turbo-FSK symbol over the duration of the transmission time interval. If $\mathbf{Y}$ (i.e.: $Y[k], \forall k \in\left[0, N_{\perp}-1\right]$ ) is the multicarrier symbol at the receiver after FFT associated to the transmitted pilot symbol $\mathbf{Z}$. Then in presence of a frequency domain complex channel $\mathbf{H}$ and frequency domain additive white Gaussian noise, $\mathbf{N}$ :

$$
\mathbf{Y}=\mathbf{Z H}+\mathbf{N}
$$

where $\mathbf{Z}$ is the the $\mathrm{ZC}$ sequence and $\mathbf{N}$ is an additive white Gaussian noise. The least square estimate of the channel $\hat{\mathbf{H}}$ is given by:

$$
\hat{\mathbf{H}}=\mathbf{H}+\left(\mathbf{Z}^{H} \mathbf{Z}\right)^{-1} \mathbf{Z}^{H} \mathbf{N}
$$

where operator ${ }^{H}$ is the hermitian transpose. We will note $\tilde{\mathbf{N}}$ the white Gaussian noise colored by the ZC sequence, $\mathbf{Z}$ :

$$
\tilde{\mathbf{N}}=\left(\mathbf{Z}^{H} \mathbf{Z}\right)^{-1} \mathbf{Z}^{H} \mathbf{N}
$$

Improved estimation of the channel coefficients $\mathbf{H}[f]$ may be derived from the observation of $\hat{\mathbf{H}}[f]$ using a filter $W_{f}$ designed to reject $\tilde{\mathbf{Z}}$. To construct the filter $\mathbf{W}_{\mathbf{f}}$, the following minimization problem should be solved:

$$
\arg \min _{\mathbf{W}_{\mathbf{f}}} E\left[\left\|\mathbf{W}_{\mathbf{f}} \hat{\mathbf{H}}-\mathbf{H}\right\|^{2}\right]
$$

Let $\Omega$ be defined as:

$$
\Omega=E\left[\left\|\mathbf{W}_{\mathbf{f}} \hat{\mathbf{H}}-\mathbf{H}\right\|^{2}\right]
$$

Using (6) and (7), (9) may then be rewritten as:

$$
\begin{aligned}
\Omega=E[\| & \\
=E\left[\begin{array}{l}
\mathbf{f} \\
=E
\end{array}\right. & \mathbf{W}_{\mathbf{f}} \mathbf{H} \mathbf{H}^{H} \mathbf{W}_{\mathbf{f}}{ }^{H} \\
& -\mathbf{W}_{\mathbf{f}} \mathbf{H} \mathbf{H}^{H}-\mathbf{H} \|^{H} \mathbf{W}_{\mathbf{f}}{ }^{H} \\
& \left.\left.+\mathbf{H} \mathbf{H}^{H}+\mathbf{W}_{\mathbf{f}} \tilde{\mathbf{N}} \tilde{\mathbf{N}}^{H} \mathbf{W}_{\mathbf{f}}{ }^{H}\right]\right]
\end{aligned}
$$

Then, if $\mathbf{h}$ is the $N \times 1$ vector of the channel impulse response, $\mathbf{H}$ may be written as:

$$
\mathbf{H}=\mathbf{F}\left(\mathbf{1}: \mathbf{N}_{\perp},:\right) \mathbf{h}=\mathbf{F}_{\mathbf{N}_{\perp}} \mathbf{h}
$$

Finally (9) may be rewritten as:

$$
\begin{aligned}
\Omega=t r & {\left[\mathbf{W}_{\mathbf{f}} \mathbf{F}_{\mathbf{N}_{\perp}} \boldsymbol{\Phi}_{\mathbf{h}} \mathbf{F}_{\mathbf{N}_{\perp}}{ }^{H} \mathbf{W}_{\mathbf{f}}{ }^{H}-\mathbf{W}_{\mathbf{f}} \mathbf{F}_{\mathbf{N}_{\perp}} \boldsymbol{\Phi}_{\mathbf{h}} \mathbf{F}_{\mathbf{N}_{\perp}}{ }^{H}\right.} \\
& -\mathbf{F}_{\mathbf{N}_{\perp}} \mathbf{\Phi}_{\mathbf{h}} \mathbf{F}_{\mathbf{N}_{\perp}}{ }^{H} \mathbf{W}_{\mathbf{f}}{ }^{H}+\mathbf{F}_{\mathbf{N}_{\perp}} \boldsymbol{\Phi}_{\mathbf{h}} \mathbf{F}_{\mathbf{N}_{\perp}}{ }^{H} \\
& \left.+\mathbf{W}_{\mathbf{f}} \mathbf{R}_{\tilde{\mathbf{N}}} \mathbf{W}_{\mathbf{f}}{ }^{\mathbf{H}}\right]
\end{aligned}
$$

where $\boldsymbol{\Phi}_{\mathbf{h}}$ is the time domain channel autocorrelation matrix of size $N \times N$ and $\mathbf{R}_{\tilde{\mathbf{N}}}$ is the autocorrelation matrix of the noise colored by the pilot sequence. By taking the partial derivative of $\Omega$ with respect to $\mathbf{W}_{\mathbf{f}}$ and making it equal to zero, (12) becomes:

$$
\begin{aligned}
& \frac{\partial \Omega}{\partial \mathbf{W}_{\mathbf{f}}}=0 \\
& 0 \quad=\left(\boldsymbol{\Delta}+\mathbf{R}_{\tilde{\mathbf{N}}}\right) \mathbf{W}_{\mathbf{f}}{ }^{H}-\boldsymbol{\Delta} \\
& \mathbf{W}_{\mathbf{f}}{ }^{H}=\left(\boldsymbol{\Delta}+\mathbf{R}_{\tilde{\mathbf{N}}}\right)^{+} \boldsymbol{\Delta}
\end{aligned}
$$

where $\boldsymbol{\Delta}=\mathbf{F}_{\mathbf{N}_{\perp}} \boldsymbol{\Phi}_{\mathbf{h}} \mathbf{F}_{\mathbf{N}_{\perp}}{ }^{H}$. When pilots with constant amplitude are sent then the noise distribution after LS estimation is the same for each sample and therefore $\mathbf{R}_{\tilde{\mathbf{N}}}=\sigma_{\mathbf{n}}^{2} \mathbf{I}$ with $\sigma_{n}^{2}$ the variance of the noise. However, if a modified frequency ZC sequence (as defined in (3)) are transmitted, then the amplitude of each pilot differs from one to another. Consequently the statistic of the noise after LS estimation varies and in that case, $\mathbf{R}_{\tilde{\mathbf{N}}}=\sigma_{\mathbf{n}}^{\mathbf{2}} \mathbf{R}_{\mathbf{N Z}}$ where $\mathbf{R}_{\mathbf{N Z}}(i, j)=0$ when $i \neq j$, $\|\mathbf{Z}(i)+\mathbf{X}(i)\|^{-2}$ otherwise.

The question, then, is how the performance of the filter is impacted by the introduction of the interference added to limit PAPR in the case of the modified ZC sequence. Therefore performance of the proposed channel estimation scheme has been evaluated and compared to the performance of the time $\mathrm{ZC}$ sequence. Results of the comparison are given in the next section.

\section{ESTIMATED PERFORMANCE}

\section{A. Channel estimation performance}

The performance of the proposed filtered Least Square algorithm has been evaluated for both $\mathrm{ZC}$ sequences of (1) and (3). The size of the FFT, $N$, has been assumed to be equal to 512 points and $N_{\perp}$ is set to 32 . The relative mean square error (RMSE) of the estimated channel coefficients is evaluated for various values of SNR assuming perfect knowledge of the channel statistics (i.e. $\boldsymbol{\Phi}_{\mathbf{h}}$ ). Results show almost identical 


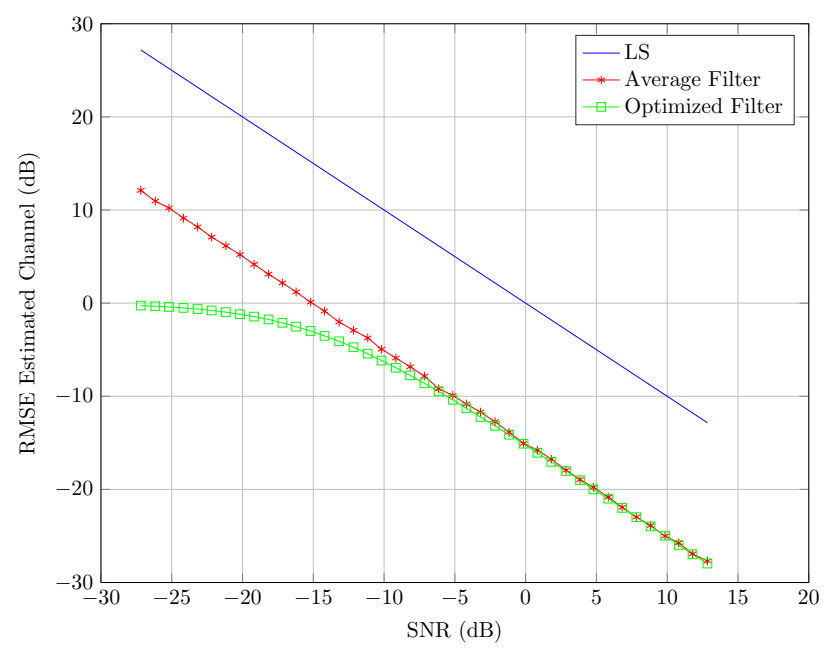

Fig. 4. Performance of channel estimation under AWGN channel.

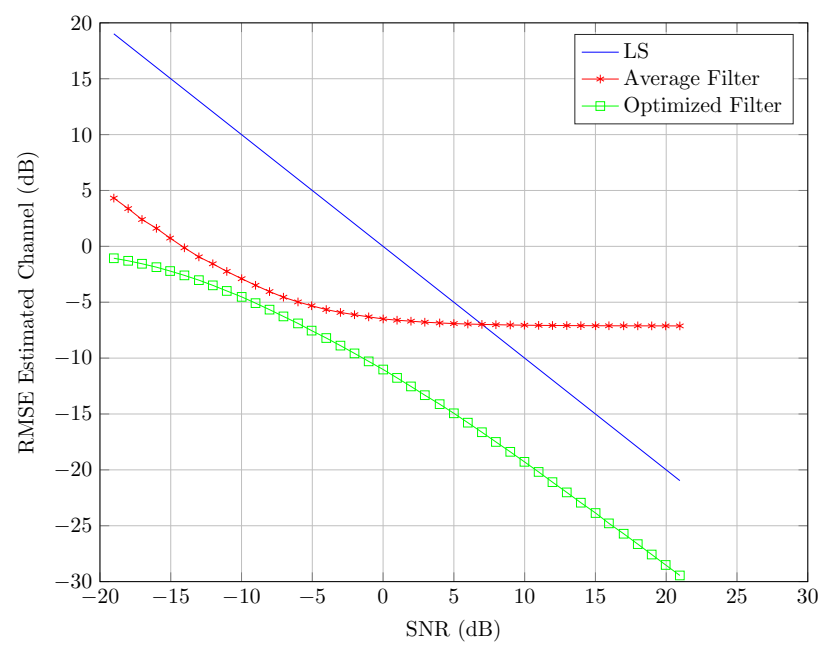

Fig. 5. Performance of channel estimation under ETU channel.

performance level between time $\mathrm{ZC}$ sequence and modified frequency $\mathrm{ZC}$ sequence. Fig. 4 gives the performance of the channel estimation method in presence of AWGN channel. The performance of the average filter is also given and is optimal in this case as the channel is a constant value. The gain of performance is significantly better than the LS approach without any filtering. In this case, an asymptotic gain of $10 \log \left(N_{\perp}\right)$ or $15 d B$ is observed. The performance degradation introduced by the noise coloring of the modified frequence $\mathrm{ZC}$ sequence has been evaluated to be less than $0.2 \mathrm{~dB}$ and is considered negligible.

Similarly, channel estimation performance is evaluated for the Extended Typical Urban (ETU) channel model of 3GPP. This channel emulates the impulse response of a signal received in a strong multipath environment and is typical of LPWA applications in a urban environment. In this case the coherence bandwidth is smaller than the bandwidth of the 32 active carriers (carrier spacing was $15 \mathrm{kHz}$ ), hence the average filter is, as expected, inefficient as channel coefficients are not constant. Gain introduced by $\mathbf{W}_{\mathbf{f}}$ filter is approximately $10 \mathrm{~dB}$ in comparison to the LS case.

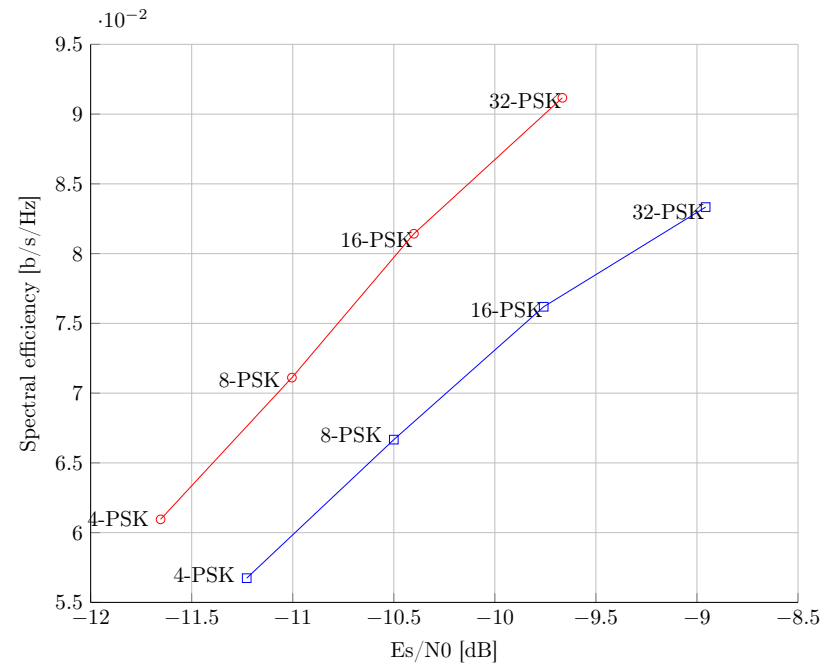

Fig. 6. AWGN performance of Turbo-FSK with $N_{\perp}=32$ sub-carriers and $N_{L}$-PSK, $N_{L}=\{4,8,16,32\}, \lambda=4$ to reach a PER of $10^{-2}$. Assuming perfect channel estimation. (red - block length equals to 1024 bits - blue block length equals to $256 \mathrm{bits})$

\section{B. Performance loss introduced by imperfect channel estima- tion}

In order to evaluate the performance loss introduced by the proposed channel estimation scheme and the requirements put on the pilot period $D$ as defined in Section II, performance of Turbo-FSK has been simulated under AWGN Channel (Fig. 6) for various values of $N_{L}$ from 4 to 32 with $\lambda=4$ and different payload lengths (256bits and 1024bits). This configuration has been selected as typical for LPWA applications. As expected, as the constellation order $N_{L}$ is increased, spectral efficiency is also increased and the required level of SNR to reach a packet error rate (PER) less than $10^{-2}$ is also increased. Furthermore, SNR requirement to attain performance is lower when longer packets are transmitted. SNR interval of interest is between -12 and $-9 \mathrm{~dB}$ and well below $0 \mathrm{~dB}$.

Then, for the same configurations, the performance loss introduced by a non-perfect channel estimation is evaluated. AWGN is added to the perfect channel state information of the received Turbo-FSK symbols and associated performance loss as a function of the added noise on the channel state information is measured by simulation. We defined as $R M S E_{H}$, the amount of AWGN power that has been added. $R M S E_{H}$ is varied from $-5 \mathrm{~dB}$ to $-20 \mathrm{~dB}$. Results can be found in Fig. 7 for a block length of 256-points and in Fig. 8 for a block length of 1024-points.

When $R M S E_{H}$ is lower than $-10 \mathrm{~dB}$, performance loss may almost be approximated to an extra source of additive white noise. For instance, when $R M S E_{H}$ is equal to $-10 \mathrm{~dB}$, performance loss introduced by an extra uncorrelated noise source should be of around $10 \log \left(1+10^{-\frac{10}{10}}\right)=0.4 \mathrm{~dB}$. This first order approximation proves relatively correct for lower FSK constellations (4-PSK ans 8-PSK). However, performance loss is significantly larger for higher values of $N_{L}$ (16-PSK and 32-PSK). Furthermore, when $R M S E_{H}$ is larger than $-10 \mathrm{~dB}$, significant performance degradation is observed. For data frames with a shorter payload (256bits), performance loss ranges from $1.5 \mathrm{~dB}$ for lower values of $N_{L}$ up to $3.3 \mathrm{~dB}$ for 


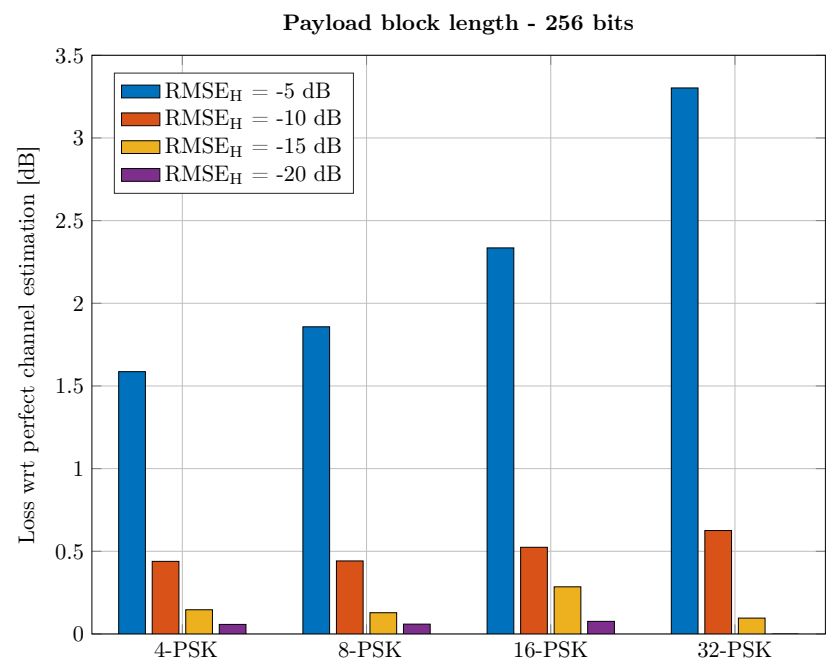

Fig. 7. Performance loss introduced by imperfect channel estimation. Payload block length 256 bits.

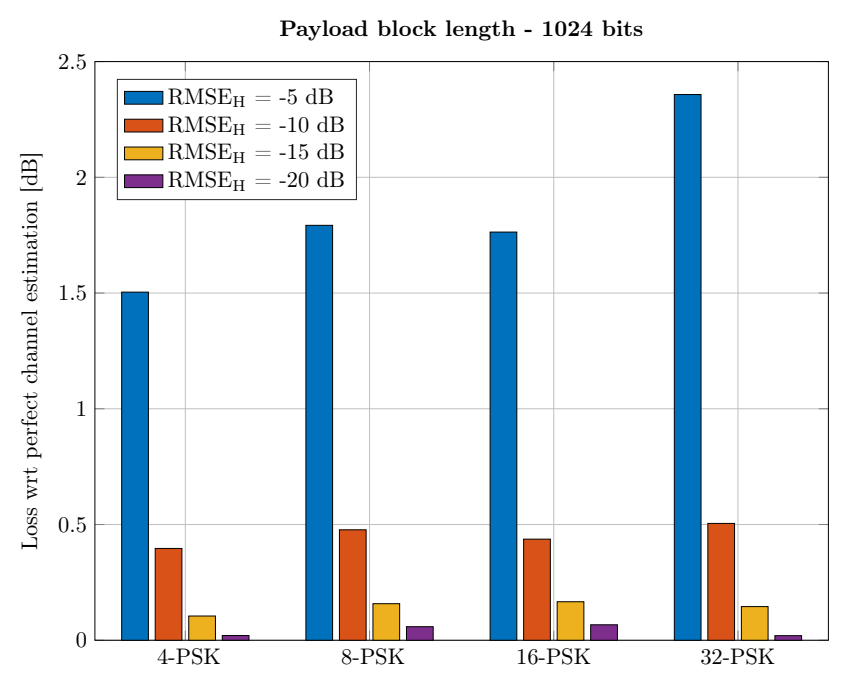

Fig. 8. Performance loss introduced by imperfect channel estimation. Payload block length 1024bits.

larger values of $N_{L}$.

\section{Recommended pilot structure}

These results (from Fig. 4 to Fig. 8) help derive some recommendations for channel estimation as a function of the performance loss budget allocated to channel estimation. For Turbo-FSK, performance is obtained for values of SNR between $-11.5 \mathrm{~dB}$ and $-9 \mathrm{~dB}$. For these levels of SNR, the channel estimation of the filtered LS algorithm gives a RMSE close to $-5 \mathrm{~dB}$ when AWGN channel is considered (Fig. 4). Further noise rejection is thus necessary if performance loss incurred by channel estimation need to be contained below $1.5 \mathrm{~dB}$ (Fig. 7 and Fig. 8). This can be achieved by interpolating the resulting channel estimation across multiple pilot symbols (time domain interpolation). Hence, channel time interpolation should not only estimate channel variations between pilot symbols but also filter unwanted noise introduced by imperfect channel estimation. To obtain $R M S E_{H}$ levels of $-10 \mathrm{~dB}$ (resp. $-15 \mathrm{~dB}$ ) interpolation over at least 3 pilot symbols (resp. 4) should be performed to avoid significant performance degradation.

\section{CONCLUSION}

Turbo-FSK is a waveform that is well adapted to future LPWA systems for 5G systems: its performance is very close to Shannons limit and furthermore the waveform exhibits a constant envelope. However, the impact of channel estimation has so far not been thoroughly considered on the performance of the turbo-FSK receiver.

This paper discussed and evaluated a channel estimation technique based on distributed pilot symbols. A pilot sequence based on a frequency domain ZC sequence has been studied and optimized for PAPR reduction. The PAPR of the pilot sequence has been reduced to below $1 \mathrm{~dB}$ and the performance of channel estimation has been studied. Impact on overall performance of the receiver has been evaluated particularly for the very low levels of SNR that characterize the selected LPWA scenario.

\section{ACKNOWLEDGMENT}

The research leading to these results was supported by the French Agence Nationale de la Recherche (ANR), under grant agreement ANR-16-CE25-0002 (project EPHYL).

\section{REFERENCES}

[1] L. Columbus. 2017 Roundup of Internet of Things Forecasts. Forbes, 2018. [Online]. Available: https://www.forbes.com/sites/louiscolumbus/2017/12/10/2017roundup-of-internet-of-things-forecasts/\#66ae9cf71480. [Accessed: Oct-2018]

[2] F. H. Raab, P. Asbeck, S. Cripps, P. B. Kenington, Z. B. Popovic, N. Pothecary, J. F. Sevic, and N. O. Sokal, "Power amplifiers and transmitters for $\mathrm{rf}$ and microwave," IEEE Transactions on Microwave Theory and Techniques, vol. 50, no. 3, pp. 814-826, March 2002.

[3] S. L. Miller and R. J. O'Dea, "Peak power and bandwidth efficient linear modulation," IEEE Transactions on Communications, vol. 46, no. 12, pp. 1639-1648, Dec 1998.

[4] 3GPP, "Evolved Universal Terrestrial Radio Access (E-UTRA); Physical channels and modulation," 3rd Generation Partnership Project (3GPP), Technical Specification (TS) 36.211, 03 2017, version 14.2.0.

[5] Y. Roth, J.-B. Doré, L. Ros, and V. Berg, "Turbo-FSK: A New Uplink Scheme for Low Power Wide Area Networks," in 2015 IEEE 16th International Workshop on Signal Processing Advances in Wireless Communications (SPAWC), Stockholm, Sweden, June 2015, pp. 81-85.

[6] Y. Roth, "The physical layer for low power wide area networks : a study of combined modulation and coding associated with an iterative receiver,' Theses, Université Grenoble Alpes, July 2017. [Online]. Available: https://hal.archives-ouvertes.fr/tel-01568794

[7] L. Bahl, J. Cocke, F. Jelinek, and J. Raviv, "Optimal decoding of linear codes for minimizing symbol error rate (corresp.)," IEEE Transactions on Information Theory, vol. 20, no. 2, pp. 284-287, March 1974.

[8] Y. Roth, J.-B. Doré, L. Ros, and V. Berg, A Comparison of Physical Layers for Low Power Wide Area Networks. Springer International Publishing, 2016.

[9] F. Dehmas, V. Mannoni, and V. Berg, "Turbo-FSK, a physical layer for LPWA: Synchronization and channel estimation," in 2018 European Conference on Networks and Communications (EuCNC), June 2018, pp. $1-5$.

[10] D. Chu, "Polyphase codes with good periodic correlation properties (corresp.)," IEEE Transactions on Information Theory, vol. 18, no. 4, pp. 531-532, July 1972. 